\title{
1 A stable pollination environment limits current but not potential evolution of \\ 2 floral traits
}

3 Maria Clara Castellanos ${ }^{1,2}$, Javier Montero-Pau ${ }^{3,4}$, Peio Ziarsolo ${ }^{3}$, Jose Miguel Blanca ${ }^{3}$, Joaquin

4 Cañizares $^{3}$, Juli G. Pausas ${ }^{2}$

5

$6{ }^{1}$ School of Life Sciences, University of Sussex, Brighton, BN1 9QG, UK

$7 \quad{ }^{2}$ CIDE-CSIC, Montcada, Valencia, Spain

$8{ }^{3}$ COMAV, Universitat Politècnica de València, Valencia, Spain

$9{ }^{4}$ Department of Biochemistry and Molecular Biology, Universitat de València, Valencia, Spain

11 Corresponding author: Maria Clara Castellanos m.c.castellanos@sussex.ac.uk

13 Running title: Floral evolutionary potential

\section{Abstract}

Plants' vast variation in floral traits at a macroevolutionary level is often interpreted as the result of adaptation to pollinators. However, field studies often find no evidence of pollinator-mediated selection on flowers. This could be explained by periods of stasis, when selection is relaxed under stable conditions, followed by pollinator changes that provide innovative selection. We asked if periods of stasis are caused by stabilizing or absence of other forms of selection on floral traits, or by low trait heritability even if selection is present. We studied Ulex parviflorus, a plant predominantly pollinated by one bee species across its range. We measured heritability and evolvability of floral traits, using genome-wide molecular relatedness in a wild population, and combined this with estimates of selection. We found evidence for both stabilizing selection and low trait heritability as explanations for stasis in flowers. The area of the standard petal is under stabilizing selection, but the variability observed in the wild is not heritable. A separate trait, floral size, in turn presents high heritability, but is not currently under selection. We show how a stable pollination environment can lead to a lack of evolutionary change, yet maintain heritable variation to respond to future selection pressures. 


\section{Introduction}

Flowering plants exhibit a striking diversity in floral form and function, and because flowers are reproductive organs, the causes and dynamics of their evolution are crucial for understanding plant biodiversity. Much of the variation in floral traits at a macroevolutionary level is often interpreted as the result of adaptations to pollinators (Fenster et al. 2004). Experimental studies also confirm that many floral traits can be subject to selection by pollinators (reviewed by Parachnowitsch and Kessler 2010, Caruso et al. 2018). However, field studies measuring pollinator-mediated evolution of floral traits often find sporadic and erratic evidence for strong selection taking place in wild populations (Harder and Johnson 2009). In their review, Harder and Johnson found that only about $1 / 3$ of the studies reported significant selection on floral traits. A possible reason for this 'paradox' is the likely prevalence of periods of stasis, where pollinator-mediated selection on flowers is relaxed under stable conditions, interrupted by more unstable periods where pollinator changes can provide innovative selection (e.g. Galen 1989, Harder and Johnson 2009).

For pollinator-mediated evolution to take place in the wild, floral phenotypic traits must not only be under selection but also harbour enough heritable variation. Periods of stasis can thus be the consequence of stabilizing or a lack of directional/disruptive selection on traits, or alternatively, they can also be the result of low levels of heritable variation even if selection is present. An appropriate model to study the role of these two non-exclusive scenarios would be a plant with a stable single dominant pollinator. Under these stable conditions, floral traits can be expected to experience low levels of pollinator-driven innovative selection, but still be heritable. Heritable variation in floral traits has been shown for numerous species in the greenhouse (reviewed in Ashman and Majetic 2006, Opedal 2018), and in a few field studies (Schwaegerle and Levin 1990, Mazer and Schick 1991, Campbell 1996, Galen 1996). Thus a relaxation of selection could be the most likely explanation for stasis in floral traits in populations with stable pollination environments.

However, it also possible that trait heritability is lower in wild conditions than indicated by estimates under artificially reduced environmental variation. Traditional greenhouse and common garden studies of heritability allow for control of local environments and genetic background, but heritability values measured under controlled conditions can be systematically overestimated compared with wild conditions (Conner et al. 2003, Winn 2004). This can be caused by higher environmental variability in the field, as well as decreased expression of additive variance, or potential differences in survival, all leading to smaller heritability estimates. The alternative of 
measuring heritability directly in the field, although being more realistic, was until recently constrained by difficulties in designing complex crossing and planting experiments (see Campbell 1996), or in establishing relatedness among individual plants growing in the wild. This has now changed thanks to access to large and highly informative molecular markers (Castellanos et al. 2011, Stanton-Geddes et al. 2013). Using genome-wide markers to measure genetic similarity of plants growing in the wild (in the form of a relatedness matrix, $G$ ), it is possible to estimate the proportion of the phenotypic covariance that is explained by relatedness (i.e. heritability) in the focal floral trait (Ritland 1996). This approach can incorporate environmental factors in the statistical estimation of heritability, to provide us with an ecologically realistic view of what plant populations are experiencing in natural conditions and help us understand the role of genetic variation in evolution (Campbell 1996, Kruuk et al. 2014).

We study the consequences of a stable pollination environment on floral traits by focusing on a plant with a dominant pollinator, the Mediterranean gorse (Ulex parviflorus). Observations across its current distribution show that honey bees (Apis mellifera) are currently the prevailing pollinator, with consistently low visitation rates, including in areas with low human influence. Dominance of honey bee visitation was observed by Herrera (1988; no \% of visits reported) and Reverté et al. (2016; 63\% of visits were by honey bees) in coastal populations in southern and eastern Spain respectively, and has also been observed in inland populations in Cazorla, Spain (93\% of visits; C.M. Herrera pers com.). Pollinator-mediated selection on flowers is expected in this plant because Ulex and relatives (the large legume subfamily Faboidae) often have complex irregular butterfly-type flowers ("papilionoid" or "keel" flowers, Fig. 1) believed to be specialized on bee pollination, with traits that both enhance pollinator attraction and mechanical interactions that improve pollination (Westerkamp 1997). In such system, we predict 1) a relaxation of directional or disruptive (i.e. innovative) selection of floral traits, and/or 2) low trait heritability as a consequence of genetic erosion over time.

To test these predictions, we measured trait heritability and natural selection on the same plant individuals in a wild population; this allowed us to assess the potential for evolution in response to current and future selection. To our knowledge, this is the first time this approach is used successfully to study floral traits. We measured floral morphology and pollinator visits, along with natural selection, genetic correlation, evolvability, and heritability of the floral traits to 1 ) determine if floral traits in a stable pollination environment are currently under selection and show heritable 
102

103

104

105

106

107

108

109

110

111

112

113

variation, thus evolving in response to selection, and 2) if not, to establish if the causes for a lack of evolutionary response are related to low selection, low heritability or both.

\section{Materials and Methods}

\section{Study species and sampling locations}

Ulex parviflorus Pourr. (Mediterranean gorse; Fabaceae) is a thorny perennial shrub that lacks true leaves in the adult stage and grows up to $2 \mathrm{~m}$. Species in the genus Ulex have yellow hermaphroditic flowers visited and pollinated by large-bodied bees, in a similar way to other species in the tribe Genistae (Herrera 2001). Flowers do not produce nectar and the bees visit to collect pollen, but to be able to do so, they need to be heavy enough to actively trigger the explosive mechanism for pollen release. Reproductive organs in these flowers are enclosed by specialized petals, the keel and the wings (Fig. 1). The insect presses the keel petals with the hind legs and this pressure powerfully releases the concealed stamens and stigma upwards, placing a cloud of pollen grains on the ventral side of the bee. After a visit flowers do not recover their original shape, with stigmas and style now protruding from the keel, and are rarely visited by large bees again, but can receive visits by smaller insects like hoverflies and solitary bees. Ulex parviflorus is self-com patible but depends on pollinators to set fruit (Herrera 1987). Flowering starts in the winter and can last for a few months into the spring.

The species is widespread along the western Mediterranean coast from southern France to southern Portugal. It is a successful colonizer of oldfields resulting from abandoned human activities, as well as recently burnt areas, thanks to numerous adaptations to recruitment after fire (Pausas et al. 2012, Pausas and Moreira 2012, Pausas et al. 2017). The seeds form a persistent bank in the soil, where they remain dormant until the heat produced during a fire breaks dormancy and stimulates germination in post-fire conditions (Moreira et al. 2010). Current landscapes in eastern Spain are a mosaic of oldfields and postfire shrubland (Pausas \& Millán 2019), where Ulex parviflorus is very abundant and distributed continuously from the lowlands up to $900 \mathrm{~m}$ of altitude (Fig S1 in supplementary materials). As a consequence there is very low genetic differentiation in the study area (Moreira et al. 2014; see also Supplementary methods and Fig. S3), and different stands cannot be considered distinct populations. For our sampling, we selected six sites within this continuous population, aiming to capture the variability of mature U. parviflorus stands in the area (Table S1, Fig. S1). By sampling at different altitudes, for example, we include variability in floral traits along the elevational gradient (Fig. S2 in supplementary materials). At each site, we tagged 40 individual plants 
136 (240 plants in total) for phenotypic and genotypic characterization as described below. Individuals were at least $5 \mathrm{~m}$ apart from each other and blooming at the time of sampling.

\section{Pollinator censuses}

To quantify the diversity of floral visitors and visitation rates, we ran multiple three-minute long pollinator censuses at different times of the day, for up to five hours of observations per locality, on two separate days during peak blooming in 2014. We also ran censuses in two localities in 2013, again during peak blooming (Montserrat and Cheste). Each census recorded the number and identity of visitors to patches of flowers on haphazardly chosen individuals. We counted the number of flowers included in each census to estimate the per-flower visitation rate.

\section{Floral phenotypes}

We collected five haphazardly selected flowers from each individual plant for phenotypic characterization of two floral traits that function as proxies for flower showiness and flower size. The area of the upwards-facing petal, or standard, plays a key role in flower showiness, as it is the largest and more visible petal in these typical papilionoid flowers (Fig.1; standard petals are also often called flag or banner petals). We removed standards from all flowers when fresh, pressed them flat individually in a plant press until dry. We then used scanned images of the standards to measure their surface area with the Image-J analysis freeware (Schneider et al. 2012).

Flower size is important in the Genistae as it can determine the size of the insects that can visit the flowers (Herrera 2001, Córdoba and Cocucci 2011). Size was estimated as the dry weight of flowers (calyx and corolla) after removing the standard petal and the pedicel, and carefully brushing off all pollen grains. Flowers were pressed and oven-dried at $40^{\circ} \mathrm{C}$ for 48 hours and weighed to the nearest $0.01 \mathrm{mg}$.

These traits were chosen because they can be expected to play an important role in the interaction with pollinators and thus be under natural selection driven by pollinators (see Study Species above). As is the case in many complex flowers, the two traits studied can be expected to co-vary (Herrera 2001), and analyses below are designed to take this into consideration. We have no reason to suspect that there is variability in these traits with flower age (see also Herrera 2001). We have never observed florivory in this species and thus doubt that herbivores will directly select for the two focal traits in this study. 


\section{Plant genotyping}

171 Fresh terminal twigs were collected from each tagged individual plant and dried in silica gel previous

172 to DNA extraction. The extraction was performed using the Speedtools plant DNA extraction kit

173 (Biotools, Madrid, Spain), with modifications to the manufacturer's protocol to optimize DNA

174 quantity and quality extracted for this highly lignified species. We used the Genotyping-by-

175 Sequencing (GBS) protocol to identify single nucleotide polymorphisms (SNPs) across the genome

176 (Elshire et al. 2011). Illumina libraries for our 240 individuals were constructed by digesting genomic

177 DNA with a restriction enzyme. The GBS protocol was followed twice for each plant after separate

178 digestions with Pstl and ECoT22I, in order to increase the number of high quality SNPs. Library

179 construction and sequencing was performed by the Genomic Diversity facility at Cornell University

180 (USA). SNP calling was implemented using the UNEAK pipeline (Lu et al. 2013) in the TASSEL v.3

software package (Bradbury et al. 2007), designed for data sets without a reference genome.

The final SNP dataset used for the analysis of relatedness below excluded loci that were not genotyped in at least $90 \%$ of individual plants. The minimum allele frequency allowed to retain loci was set to MAF > 0.01 . We also excluded individuals with low genotyping rates (under $85 \%$ of loci). After applying these filters, we also manually removed remaining loci with extreme values of observed heterozygosity (under $2 \%$ and higher than $98 \%$ ), after estimating oHET with PLINK command-Hardy (Purcell et al. 2007).

\section{Fitness estimates and phenotypic selection}

We estimated fruit set in the same 40 individual plants in each locality as a proxy for female reproductive success. For this, we labelled a representative flowering twig in each plant during flowering peak. When fruits were already developing (browning capsules) a few weeks later, we collected the labelled twig in a paper envelope. Back in the laboratory we measured $10 \mathrm{~cm}$ of twig to calculate a) the number of fruits developing normally, and b) scars left by all flowers produced by the twig, clearly visible under a dissecting microscope. From this we calculated fruit set as the proportion of flowers that develop into a fruit. The majority of fruits had one ( $71 \%$ of 3200 fruits examined) or two seeds (25\%), with a mean number of 1.22 seeds/fruit across all individuals.

We estimated selection parameters to test for both linear and non-linear selection on the two floral traits, using fruit set as the response fitness variable in the models. Because floral weight and standard area show a significant phenotypic correlation (even though floral weight did not include the standard, Pearson $r=0.43, \mathrm{P}<0.001$ ), we estimated selection gradients in addition to selection 
204

205

206

207

208

209

210

211

212

213

214

215

216

217

218

219

220

221

222

223

224

225

226

227

228

229

230

231

232

233

234

235

236

237

differentials. Selection differentials provide univariate estimates of selection without considering other traits, while gradients provide estimates on correlated traits. By estimating the four selection parameters - standardized linear (S), and quadratic (c) selection differentials, and standardized linear $(\beta)$ and quadratic $(\gamma)$ selection gradients - we can explore direct and indirect selection on the floral traits. Linear parameters test for directional selection, while quadratic parameters measure potential stabilizing or disruptive selection.

We used generalised additive models (GAM) to measure selection parameters on absolute fitness values, following the approach developed by Morrisey and Sakreda (2013). This approach provides quantitative estimates of selection differentials and gradients for non-normal fitness components, testing for both linear and quadratic selection. We fitted GAMs for binomial fruit set data (fruits developed in relation total flowers), using a logit link function and assuming a binomial error distribution with the mgcv package in $\mathrm{R}$. We used univariate GAMs to estimate selection differentials, and included both floral traits into a bivariate model to estimate selection gradients. To control for local effects, we included locality as a random factor in all models. Models included additive spline effects on all factors. Differential and gradient parameters were estimated based on numerical approximations of first and second partial derivatives of relative fitness, averaged over the distribution of observed phenotype. To calculate the significance of selection differentials and gradients, we used the bootstrap approach ( $n=1000$ samples) implemented in the gsg package in $R$ (Morrissey and Sakrejda 2013).

\section{SNP-based relatedness and quantitative genetic parameters}

Pairwise relatedness between all pairs of individuals was estimated from the similarity of their SNP genotypes. To estimate $\mathrm{G}$, the genome-wide relatedness matrix among all pairs of individuals, we used the realized relatedness method of VanRaden (2008) and Astle and Balding (2009) as implemented in the kin function of package synbreed in $R$ (Wimmer et al. 2012; see details in Supplementary methods). Relatedness values under this approach are a measure of excess allele sharing compared to unrelated individuals. As a consequence, negative values can be common and correspond to individuals sharing fewer alleles than expected given the sample.

To estimate additive genetic variance (and then heritability and evolvability) we used a linear mixed 'animal model' approach to model the phenotypic variance in floral traits while including the variance explained by relatedness (Wilson et al. 2010). We included the elevation above sea level as a fixed effect to account for environmental variability among plants, because elevation is the main factor that varies among localities (Fig. S2) and this could affect floral traits as in other species 
238 (Herrera 2005). In addition to the additive genetic effects (see model below), models included two

239 more random effects: the site of origin of each plant, to account for unmeasured local

240 environmental effects that could co-vary with genetic variation, and the individual identity to

241 account for intra-individual effects (a "permanent environment" effect in Wilson et al. 2010),

242 because we had five flower replicates per plant. We ran a univariate model for each of the two

243 floral traits studied, specified as:

$$
y=X \beta+Z_{1} a+Z_{2} s+Z_{3} i+e
$$

where $y$ is the vector of floral trait values, $\beta$ is the vector of fixed effects (with $X$ as the incidence matrix), $Z_{1}, Z_{2}$ and $Z_{3}$ are incidence matrices for the random effects $a$ (individual identity to partition additive genetic effects), $s$ (the locality), $i$ (individual identity to model intra-individual effects caused by differences among replicate flowers from the same individual), and e is the residual error. The variance-covariance structure of random factor $a$ in the model is defined by $G \cdot V_{a}$, where $\mathrm{G}$ is the genome-wide relatedness matrix between plant pairs, and $V_{a}$ is the additive variance to be estimated. To test for the effect of not including the spatial and environmental predictors in the models, we also ran a 'naive' version of each model that included only the relatedness and individual effects (Castellanos et al. 2015). We ran Bayesian animal models using package MCMCg/mm for $R$ (Hadfield 2010) with both floral weight and standard petal area modeled as continuous traits. For modelling the standard area, we used parameter expanded priors for the distribution of variance components following the $\chi^{2}$ distribution with one degree of freedom. Each analysis was iterated long enough to obtain 5000 independent chains (see supplementary methods and Table S2 for model details, scripts and prior selection).

Narrow sense heritability $\left(h^{2}\right)$ was then estimated as the proportion of the total phenotypic variance assigned to the individual (i.e. to the additive genetic variance, $V_{a}$ ):

$$
h^{2}=\frac{V_{a}}{V_{a}+V_{s}+V_{i}+V_{e}}
$$

where $V_{S}$ is the variance explained by the site of origin, $V_{i}$ is the intra-individual variance in the trait, and $V_{e}$ is the residual variance. We also estimated the narrow sense evolvability (e), i.e. the meanstandardized additive genetic variance, $e=\mathrm{V}_{a} / x^{2}$, where $x$ is the trait mean. $e$ reflects the expected percentage of change of a trait under a unit strength of selection per generation (Houle 1992, Hansen et al. 2003) and provides an estimate of evolvability that is independent of trait variation and comparable across traits. 
271 In addition, we estimated the genetic correlation $\left(r_{G}\right)$ between floral weight and standard area by

272 running a bivariate animal model in $M C M C g / m m$. In this case we used the same fixed and random

273 factors as in the univariate models above (see supplementary methods for prior information).

275 Results

\section{Pollinators}

277 We recorded 364 visits to 22522 censused flowers in 28 hours of observations across the six $U$. parviflorus localities. Of those, 331 (92\%) were visits by the honeybee Apis mellifera. Further 25 visits were by Bombus $s p$. individuals (7\%). The remaining 3 visits were to already open flowers by small coleoptera and a hoverfly, both unlikely to contact stigmas and carry out pollination. Across sites, we found an average visitation rate of $0.015( \pm 0.057)$ visits per 3-minute census to an individual flower, which translates into a visit every 3.3 hours, on average. Visitation rates were similar when comparing localities, except for one where visits were significantly more frequent (Simat average visitation rate $=0.03$ visits per census).

\section{Floral phenotypes and selection}

Flowers show considerable variation in the two traits measured, flower weight and standard petal area, both within and across localities. A variance partition analysis showed that the variance in both traits across the five flowers sampled per plant was negligible, so the selection analysis below was run using mean floral values for each individual plant (see also Herrera 2001).

We found no evidence of linear directional selection on floral traits, either in univariate models ( $\mathrm{s}$ coefficients) or models of correlated selection incorporating both floral variables ( $\beta$ coefficients, Table 1). However, we found evidence for univariate quadratic effects in both traits (c coefficients) but only standard area shows significant quadratic gradients ( $\gamma$ coefficients). This suggests that floral weight is not under direct selection, while there is strong evidence for stabilising selection on standard petal area (Fig. 2).

\section{Genomic markers and population genetic structure}

300 The GBS sequencing approach yielded a large number of polymorphic SNPs across individuals

301 (261,775 SNPs before quality filtering). After MAF and heterozygosity filtering, we retained 10,421

302 high-quality SNPs that were present in at least $90 \%$ of individuals across all localities. The analyses

303 below use this dataset to estimate genomic relatedness; however, we also tested for the effect of 304 retaining a larger number of SNPs (with presence in at least $50 \%$ of the individuals, which leads to a 
305

306

307

308

309

310

311

312

313

314

315

316

higher number of genotypes imputed by synbreed, see Supplementary methods). Analysis with this larger dataset produced the same qualitative results, suggesting that retaining more (but highly imputed) markers did not add valuable information on the relatedness among our study plants. Therefore, all analyses below use the smaller dataset with 10,421 SNPs.

\section{Heritability, evolvability and genetic correlation}

Pairwise relatedness among sampled individuals varied markedly and was overall relatively low (average values ranging from -0.09 to 0.79 , but with most values $<0.2$ ), even within locality (Fig. S4), supporting the prevalence of outcrossing in this species. The low population genetic structure and the presence of variance in relatedness provide the conditions for a reliable estimation of heritability in the field in this species (Ritland 1996).

We found significant estimates of heritability and evolvability in flower weight $\left(h^{2}=0.14, e=0.42 \%\right.$; Table 2). For standard area, our models instead detected very low additive variance, yielding very low $h^{2}$ and $e$ in this case $\left(h^{2}=0.001, e<0.001 \%\right.$; Table 2). For both traits, Deviance Information Criterion (DIC) values for the heritability naive models were larger than for the complete model (Table S2), indicating a better fit for the latter. The naïve models included only the relatedness among individuals and neither environmental nor spatial predictors, and showed estimated $h^{2}$ values substantially higher than our final estimates (Table 2).

Our bivariate analysis found a low genetic correlation between the two floral traits that is indistinguishable from zero $\left(r_{G}=0.06\right)$; in addition, credible intervals were large $(-0.139$ to 0.381$)$, so we cannot confidently support the presence of a genetic correlation.

\section{Discussion}

We provide an example of a stable pollination environment that has led to a lack of innovative selection, yet maintaining enough heritable variation for responding to possible novel selection pressures, at least in some traits. In Ulex parviflorus, we found evidence for both stabilizing selection and low trait heritability as alternative explanations for lack of evolution in flowers. Specifically, the area of the standard petal is currently under stabilizing selection, but the variability we observe in the field is not heritable. Floral weight, in turn, presents high heritability, but is not currently under selection. 
Stable pollinator communities are potentially a common feature for many plant species under even environmental conditions. For the particular case of Ulex parviflorus, current evidence shows that honey bees are the most frequent pollinators in all surveyed populations, including the one studied here and other localities (Herrera 1988, Reverté et al. 2016, C.M. Herrera pers com). Other species in the genus, including U. europaeus, U. minor and U. galli, present a higher diversity of large bees among their visitors (several species of Bombus and Andrena; Kirchner and Bullock 1999, Bowman et al. 2008, Falk 2011). The dominance of honey bees in Ulex parviflorus populations could be seen as a consequence of the large anthropogenic influence across its range; however, U. parviflorus populations in an area with low human influence and high pollinator diversity (Sierra de Cazorla, see Herrera 2018) corroborates the predominance of honey bees as pollinators of this species. Regardless of the reasons for the low pollinator diversity, our study provides evidence on how stable conditions can lead to lack of current evolution in floral traits.

On the opposite side of the spectrum, field studies that do detect pollinator-mediated directional selection on unmanipulated floral traits often focus on plants that are exposed to changing pollinators, either in different parts of the species range (Herrera et al. 2006, Anderson et al. 2010) or in hybrid contact zones where there is selection against hybridization (Campbell et al. 2018). Taken together, current evidence supports the idea that pollination-driven floral evolution takes place mostly during evolutionarily innovative periods driven by to changing pollinators.

Stabilizing selection is expected in floral traits that influence the accuracy of the flower-pollinator interaction (Cresswell 2000, Armbruster et al. 2009). It is difficult to establish how common stabilizing selection is on floral traits in wild plants, because studies do not measure non-linear selection as often as directional selection (Harder and Johnson 2009, Caruso et al. 2018). For the standard petal in Ulex, we detected stabilizing selection for intermediate surface area. The size of this "flag" petal is expected to play an important role on pollinator attraction by increasing the floral colourful display (Fig. 1), so that selection against smaller sizes is expected. Too large standard petals could be selected against if they incur a higher cost for the plant. This cost could be even higher if large standard petals are developmentally restricted to overall larger flowers; however, our genetic correlation estimates suggest that the association of standard petal area with floral size is weak. This is consistent with a previous study that carefully dissected the role of the different petals in another keel flower; in Collaea argentina, Córdoba et al. (2015) found that the standard petal is not functionally integrated with another set of floral traits that collectively regulate the enclosing mechanism of stamens and pistil. That is, the mechanics of protecting the enclosed rewards in these 
372 flowers can be independent of pollinator attraction as we expected, and selection can vary across

373 floral parts.

374

375 Floral morphological traits are often found to present heritable variation (reviewed by Ashman and

376 Majetic 2006, Opedal 2018); however, most of the studies in these reviews were performed in

377 controlled environments. Our field estimates of heritability fall within the lower range of those

378 summarized in Fig. 1 of Altman and Majetic (2006), as expected from field values compared to

379 greenhouse estimates. We found that flower weight shows significant heritability, but no detectable

380 heritability in the standard petal area. Comparing petals in papilionoid flowers, Herrera (2001) found

381 that the standard had higher phenotypic variance than other petals across Genisteae, and argued

382 that its role in pollination was smaller than for the keel petals, in a similar way as Córdoba et al.

383 (2015). This and our results suggest that this petal might be prone to high environmentally-induced

384 variation, which increases the exposure to stabilising selection, but does not lead to evolutionary

385 change.

386

Heritability estimates have been criticised as poor standardized measures of evolutionary potential

in realistic ecological settings, in part because of the covariance between environmental and genetic effects (Houle 1992, Hansen et al. 2011). In this study, we estimate heritability directly in the field, statistically controlling for environmental variation, and in the same individuals used to estimate natural selection. In this context, field heritability estimates provide a very useful approach to understand the current evolutionary potential at the population level, precisely because we are interested in the role of environmental effects on the phenotypic variance, as exposed to natural selection. An alternative measure of evolutionary potential, evolvability, uses the mean of trait values to standardize the additive genetic variance and provides a comparable estimate of proportional change in a trait value after selection (Hansen et al. 2003). Our estimates of evolvability here confirm our findings in heritability, also showing near-zero evolutionary potential for the standard petal area, but higher values for flower weight. In the latter case, evolvability is estimated to be significant but small (under $1 \%$ of the trait mean value), suggesting that change in this trait would not be fast unless submitted to strong selection. This value of evolvability is within the range of evolvability values estimated for floral size specifically across plant species, as summarised in a recent review (Opedal 2018).

403

404 Our estimate of genetic correlation between the two focal traits suffers from a low sample size to 405 run a bivariate animal model and needs to be interpreted with caution. However, the lack of a 
406

407

408

409

410

411

412

413

414

415

416

417

418

419

420

421

422

423

424

425

426

427

428

429

430

431

432

433

434

435

436

437

438

439

genetic correlation is not surprising given that we cannot detect significant additive genetic variation in one of the trait (the area of the standard petal). This does contrast with the fact that there is a significant phenotypic correlation between the two traits, but as suggested by previous studies, phenotypic correlations are not always good predictors of genetic correlations, even in highly integrated organs as flowers (Gómez et al. 2009). Again, this is consistent with the decoupling of petals found in a related species with keel flowers (Córdoba et al. 2015). It is thus possible that the phenotypic correlation is caused by shared environmental factors that affect both traits in Ulex flowers, further confirming the importance of studying evolutionary potential in field realistic conditions.

Even though we could not detect a genetic correlation between the two floral traits studied here, a caveat in our analysis is that we do not include selection on other (unmeasured) potentially correlated traits. Another potential source of problems is that Ulex flowers are hermaphroditic and thus likely subject to selection via both male and female reproductive success. Our estimates of selection here are based on fruit set alone, and we cannot rule out that the two focal traits might be under selection through male function (van Kleunen and Burczyk 2008). However, the two traits studied here can be expected to affect pollen dispersal in similar ways as pollen deposition (and thus seeds sired), because the trigger mechanism forces both male and female reproductive organs to make contact with the bees at the same time. This means that factors affecting seed set and seed sire are probably highly related in keel flowers.

This study adds to a series of recent works using large sets of molecular markers to study quantitative genetics in wild populations, mostly focused on animals (Perrier et al. 2018), but also on plants (Castellanos et al. 2015). Studies comparing the accuracy of SNP-based relatedness matrices compared to pedigrees are consistently showing that they can be very good approximations, as long as a large number of markers and a good sample of individuals is available (Bérénos et al. 2014, Perrier et al. 2018). This is therefore an exciting time for studying the evolution of traits directly in the wild, because field-based estimates of evolutionary potential provide new avenues to understand basic evolutionary questions (such as stasis and the role of plasticity in trait variation), but also the potential for wild organisms to respond to new selection pressures including those imposed by anthropogenic environmental change. In the specific case of flowers, our findings suggest that low-diversity pollination environments as those caused by anthropogenic pollination declines can lead to reduced selection pressures, reduced opportunity for selection, and stasis (Caruso et al. 2018), while exposure to new pollinators can lead to novel evolutionary change. 


\section{Conclusion}

441 Relative stasis can be prevalent in contemporary populations, yet heritable phenotypic variance can

442 be present; in combination with potential genetic correlations, this provides the potential to

443 respond to novel selection. Selection on floral traits is not restricted to pollinators, as herbivores and

444 abiotic factors can also be agents of selection (reviewed by Caruso et al. 2018). Regardless of the

445 source of selection, our findings contribute to explain the macroevolutionary patterns of floral

446 evolution where novel phenotypes are ubiquitous (exceptions are often related to very generalised

447 pollination that is stable over evolutionary time, see Vasconcelos et al. 2019). Populations can

448 experience stable conditions with undetectable innovative selection, but at the same time harbour

449 genetically based variability to evolve under new conditions.

\section{Acknowledgements}

452 We are grateful to Abel Rubira, Santiago Donat-Caerols, Yedra García, Eva Sánchez, Paula Cassá and

453 Lucia Tortajada for valuable help in the field. S. Donat-Caerols also provided help with genomic data

454 analysis. Jorge Sellés helped with DNA extractions. We thank C.M. Herrera for sharing unpublished

455 pollination data. Dr J. Brines kindly allowed access to his land in Simat. Financial support came from

456 projects TREVOL and FILAS (CGL2012-39938, CGL2015-64086) from the Spanish Government. MCC

457 was partly supported by the European Union's Horizon 2020 programme under the Marie

458 Sklodowska-Curie grant agreement No 706365.

\section{References}

Anderson, B., R. Alexandersson, and S. D. Johnson. 2010. Evolution and coexistence of pollination ecotypes in an African Gladiolus (Iridaceae). Evolution 64:960-972.

Armbruster, W. S., T. F. Hansen, C. Pélabon, R. Pérez-Barrales, and J. Maad. 2009. The adaptive accuracy of flowers: measurement and microevolutionary patterns. Annals of Botany 103:1529-1545.

Ashman, T., and C. Majetic. 2006. Genetic constraints on floral evolution: a review and evaluation of patterns. Heredity 96:343-352.

Astle, W., and D. J. Balding. 2009. Population structure and cryptic relatedness in genetic association studies. Statistical Science 24:451-471.

Bérénos, C., P. A. Ellis, J. G. Pilkington, and J. M. Pemberton. 2014. Estimating quantitative genetic parameters in wild populations: a comparison of pedigree and genomic approaches. Molecular Ecology 23:3434-3451.

Bowman, G., M. Tarayre, and A. Atlan. 2008. How is the invasive gorse Ulex europaeus pollinated during winter? A lesson from its native range. Plant Ecology 197:197-206.

Bradbury, P. J., Z. Zhang, D. E. Kroon, T. M. Casstevens, Y. Ramdoss, and E. S. Buckler. 2007. TASSEL: software for association mapping of complex traits in diverse samples. Bioinformatics 23:2633-2635.

Campbell, D. R. 1996. Evolution of floral traits in a hermaphroditic plant: field measurements of heritabilities and genetic correlations. Evolution 50:1442-1453. 
480

481

482

483

484

485

486

487

488

489

490

491

492

493

494

495

496

497

498

499

500

501

502

503

504

505

506

507

508

509

510

511

512

513

514

515

516

517

518

519

520

521

522

523

524

525

526

527

528

529
Campbell, D. R., A. Faidiga, and G. Trujillo. 2018. Clines in traits compared over two decades in a plant hybrid zone. Annals of Botany 122:315-324.

Caruso, C. M., K. E. Eisen, R. A. Martin, and N. Sletvold. 2018. A meta-analysis of the agents of selection on floral traits. Evolution.

Castellanos, M. C., J. M. Alcántara, P. J. Rey, and J. M. Bastida. 2011. Intra-population comparison of vegetative and floral trait heritabilities estimated from molecular markers in wild Aquilegia populations. Molecular Ecology 20:3513-3524.

Castellanos, M. C., S. C. González-Martínez, and J. Pausas. 2015. Field heritability of a plant adaptation to fire in heterogeneous landscapes. Molecular ecology 24:5633-5642.

Conner, J. K., R. Franks, and C. Stewart. 2003. Expression of additive genetic variances and covariances for wild radish floral traits: comparison between field and greenhouse environments. Evolution 57:487-495.

Córdoba, S. A., S. Benitez-Vieyra, and A. A. Cocucci. 2015. Functional modularity in a forcible flower mechanism: relationships among morphology, biomechanical features and fitness. Evolutionary ecology 29:719-732.

Córdoba, S. A., and A. A. Cocucci. 2011. Flower power: its association with bee power and floral functional morphology in papilionate legumes. Annals of Botany 108:919-931.

Cresswell, J. E. 2000. Manipulation of female architecture in flowers reveals a narrow optimum for pollen depostion. Ecology 81:3244-3249.

Elshire, R. J., J. C. Glaubitz, Q. Sun, J. A. Poland, K. Kawamoto, E. S. Buckler, and S. E. Mitchell. 2011. A robust, simple genotyping-by-sequencing (GBS) approach for high diversity species. PloS one 6:e19379.

Falk, S. J. 2011. A survey of the bees and wasps of fifteen chalk grassland and chalk heath sites within the East Sussex South Downs. Self-published, downloaded from www.bwars.com (January 2019).

Fenster, C. B., W. S. Armbruster, P. Wilson, M. R. Dudash, and J. D. Thomson. 2004. Pollination syndromes and floral specialization. Annu. Rev. Ecol. Evol. Syst. 35:375-403.

Galen, C. 1989. Measuring pollinator-mediated selection on morphometric floral traits: bumblebees and the alpine sky pilot, Polemonium viscosum. Evolution 43:882-890.

Galen, C. 1996. Rates of floral evolution: adaptation to bumblebee pollination in an alpine wildflower, Polemonium viscosum. Evolution 50:120-125.

Gómez, J., M. Abdelaziz, J. Muñoz-Pajares, and F. Perfectti. 2009. Heritability and genetic correlation of corolla shape and size in Erysimum mediohispanicum. Evolution 63:1820-1831.

Hadfield, J. D. 2010. MCMC Methods for Multi-Response Generalized Linear Mixed Models: The MCMCglmm R Package. Journal of Statistical Software 33:1-22.

Hansen, T. F., W. S. Armbruster, M. L. Carlson, and C. Pélabon. 2003. Evolvability and genetic constraint in Dalechampia blossoms: genetic correlations and conditional evolvability. Journal of Experimental Zoology 296B:23-39.

Hansen, T. F., C. Pélabon, and D. Houle. 2011. Heritability is not evolvability. Evolutionary Biology 38:258.

Harder, L. D., and S. D. Johnson. 2009. Darwin's beautiful contrivances: evolutionary and functional evidence for floral adaptation. New Phytologist 183:530-545.

Herrera, C. M. 2018. Complex long-term dynamics of pollinator abundance in undisturbed Mediterranean montane habitats over two decades. Ecological Monographs.

Herrera, C. M., M. C. Castellanos, and M. Medrano. 2006. Geographical context of floral evolution: towards an improved research programme in floral diversification. Pages 278-294 in L. D. Harder and S. C. H. Barrett, editors. Ecology and evolution of flowers. Oxford University Press.

Herrera, J. 1987. Flower and fruit biology in southern Spanish Mediterranean shrublands. Annals of the Missouri Botanical Garden 74:69-78. 
530
Herrera, J. 1988. Pollination relationships in southern Spanish Mediterranean shrublands. Journal of Ecology:274-287.

Herrera, J. 2001. The variability of organs differentially involved in pollination, and correlations of traits in Genisteae (Leguminosae: Papilionoideae). Annals of Botany 88:1027-1037.

Houle, D. 1992. Comparing evolvability and variability of quantitative traits. Genetics 130:195-204.

Kirchner, F., and J. Bullock. 1999. Taxonomic separation of Ulex minor Roth. and U. gallii Planch.: morphometrics and chromosome counts. Watsonia 22:365-376.

Kruuk, L. E., A. Charmantier, and D. Garant. 2014. The study of quantitative genetics in wild populations. Quantitative genetics in the wild:1-15.

Mazer, S. J., and C. T. Schick. 1991. Constancy of population parameters for life-history and floral traits in Raphanus sativus L. II. Effects of planting density on phenotype and heritability estimates. Evolution 45:1888-1907.

Moreira, B., M. C. Castellanos, and J. Pausas. 2014. Genetic component of flammability variation in a M editerranean shrub. Molecular ecology 23:1213-1223.

Moreira, B., J. Tormo, E. Estrelles, and J. Pausas. 2010. Disentangling the role of heat and smoke as germination cues in Mediterranean Basin flora. Annals of Botany 105:627-635.

Morrissey, M. B., and K. Sakrejda. 2013. Unification of regression-based methods for the analysis of natural selection. Evolution 67:2094-2100.

Opedal, $\varnothing$. H. 2018. The evolvability of animal-pollinated flowers: towards predicting adaptation to novel pollinator communities. New Phytologist.

Parachnowitsch, A. L., and A. Kessler. 2010. Pollinators exert natural selection on flower size and floral display in Penstemon digitalis. New Phytologist 188:393-402.

Pausas, J. G., G. A. Alessio, B. Moreira, and G. Corcobado. 2012. Fires enhance flammability in Ulex parviflorus. New Phytologist 193:18-23.

Pausas, J. G., J. E. Keeley, and D. W. Schwilk. 2017. Flammability as an ecological and evolutionary driver. Journal of Ecology 105:289-297.

Pausas J.G. \& Millán M.M. 2019. Greening and browning in a climate change hotspot: the Mediterranean Basin. BioScience 96:143-151.

Pausas, J. G., and B. Moreira. 2012. Flammability as a biological concept. New Phytologist 194:610613.

Perrier, C., B. Delahaie, and A. Charmantier. 2018. Heritability estimates from genomewide relatedness matrices in wild populations: Application to a passerine, using a small sample size. Molecular ecology resources 18:838-853.

Purcell, S., B. Neale, K. Todd-Brown, L. Thomas, M. A. Ferreira, D. Bender, J. Maller, P. Sklar, P. I. De Bakker, and M. J. Daly. 2007. PLINK: a tool set for whole-genome association and population-based linkage analyses. The American Journal of Human Genetics 81:559-575.

Reverté, S., J. Retana, J. M. Gómez, and J. Bosch. 2016. Pollinators show flower colour preferences but flowers with similar colours do not attract similar pollinators. Annals of Botany 118:249257.

Ritland, K. 1996. Marker-based method for inferences about quantitative inheritance in natural populations. Evolution 50:1062-1073.

Schneider, C. A., W. S. Rasband, and K. W. Eliceiri. 2012. NIH Image to ImageJ: 25 years of image analysis. Nature methods 9:671.

Schwaegerle, K. E., and D. A. Levin. 1990. Quantitative genetics of seed size variation in Phlox. Evolutionary ecology 4:143-148.

Stanton-Geddes, J., J. B. Yoder, R. Briskine, N. D. Young, and P. Tiffin. 2013. Estimating heritability using genomic data. Methods in Ecology and Evolution 4:1151-1158.

van Kleunen, M., and J. Burczyk. 2008. Selection on floral traits through male fertility in a natural plant population. Evolutionary ecology 22:39-54.

VanRaden, P. M. 2008. Efficient Methods to Compute Genomic Predictions. Journal of Dairy Science 91:4414-4423. 
581 Vasconcelos, T. N. C., M. Chartier, G. Prenner, A. C. Martins, J. Schönenberger, A. Wingler, and E. Lucas. 2019. Floral uniformity through evolutionary time in a species-rich tree lineage. New Phytologist 221:1597-1608.

Westerkamp, C. 1997. Keel blossoms: bee flowers with adaptations against bees. Flora 192:125-132.

Wilson, A. J., D. Réale, M. N. Clements, M. M. Morrissey, E. Postma, C. A. Walling, L. E. B. Kruuk, and D. H. Nussey. 2010. An ecologist's guide to the animal model. Journal of Animal Ecology 79:13-26.

Wimmer, V., T. Albrecht, H. Auinger, and C. Schoen. 2012. synbreed: a framework for the analysis of genomic prediction data using R. Bioinformatics 28:2086-2087.

Winn, A. A. 2004. Natural selection, evolvability and bias due to environmental covariance in the field in an annual plant. Journal of Evolutionary Biology 17:1073-1083. 
bioRxiv preprint doi: https://doi.org/10.1101/581827; this version posted June 30, 2019. The copyright holder for this preprint (which was not certified by peer review) is the author/funder, who has granted bioRxiv a license to display the preprint in perpetuity. It is made available under aCC-BY-NC-ND 4.0 International license.

592 Table 1 Directional and quadratic selection differentials and coefficients ( \pm standard errors) for the 593 two floral traits studied.

\begin{tabular}{lcccc}
\hline Trait & \multicolumn{2}{c}{ Differential } & \multicolumn{2}{c}{ Gradient } \\
\hline & Directional, $\mathbf{S}$ & Quadratic, $\mathbf{C}$ & Directional, $\boldsymbol{\beta}$ & Quadratic, $\boldsymbol{\gamma}$ \\
\hline Standard petal area & $-0.003 \pm 0.003 \mathrm{~ns}$ & $-\mathbf{0 . 0 0 2} \pm \mathbf{0 . 0 0 0 * * *}$ & $-0.041 \pm 0.039 \mathrm{~ns}$ & $-\mathbf{0 . 1 0 2} \pm \mathbf{0 . 0 2 9} * * *$ \\
Flower weight & $-0.018 \pm 0.033 \mathrm{~ns}$ & $-\mathbf{0 . 0 7 0 \pm 0 . 0 2 3 * *}$ & $-0.022 \pm 0.047 \mathrm{~ns}$ & $-0.038 \pm 0.021 \mathrm{~ns}$ \\
Interaction & & & & $0.000 \pm 0.002 \mathrm{~ns}$ \\
\hline
\end{tabular}

594 
595 Table 2 Estimates of heritability $h^{2}$ and evolvability $e$ (with $95 \%$ credibility intervals, $\mathrm{Cl}$ ) for floral 596 traits in wild Ulex parviflorus. 'Naïve' heritability models did not include spatial or environmental 597

598 predictors.

\begin{tabular}{|c|c|c|c|c|c|c|}
\hline & \multicolumn{2}{|c|}{ Naïve $h^{2}$ model } & \multicolumn{2}{|c|}{ Final $h^{2}$ model } & \multicolumn{2}{|c|}{ Evolvability } \\
\hline & $h^{2}$ & $\mathrm{Cl}$ & $h^{2}$ & $\mathrm{Cl}$ & $e$ & $\mathrm{Cl}$ \\
\hline Standard petal area & 0.76 & $0.60-0.81$ & 0.001 & $0.00-0.27$ & $<0.001 \%$ & $0.00-1.91$ \\
\hline Flower weight & 0.71 & $0.60-0.80$ & 0.14 & $0.03-0.34$ & $0.42 \%$ & $0.11-1.21$ \\
\hline
\end{tabular}




\section{$600 \quad$ Figure legends}

601

602 Figure 1. Flowers of Ulex parviflorus. (a) Flowers previous to a visit with standard petal extended and

603 reproductive organs enclosed by the keel petals and calix. (b) Pressed standard petal. (c) Flower

604 after being "triggered" by a bee visit, showing all petals and exposed reproductive organs. (Photo

605 credits: (a) MC Castellanos, (c) J. Quiles).

606

607 Figure 2. Fruit set as a function of the two floral traits measured, (a) standard petal area and (b)

608 flower weight (a proxy for floral size). Lines are generalised additive model fits with shaded areas

609 showing $95 \%$ confidence intervals. 
(a)

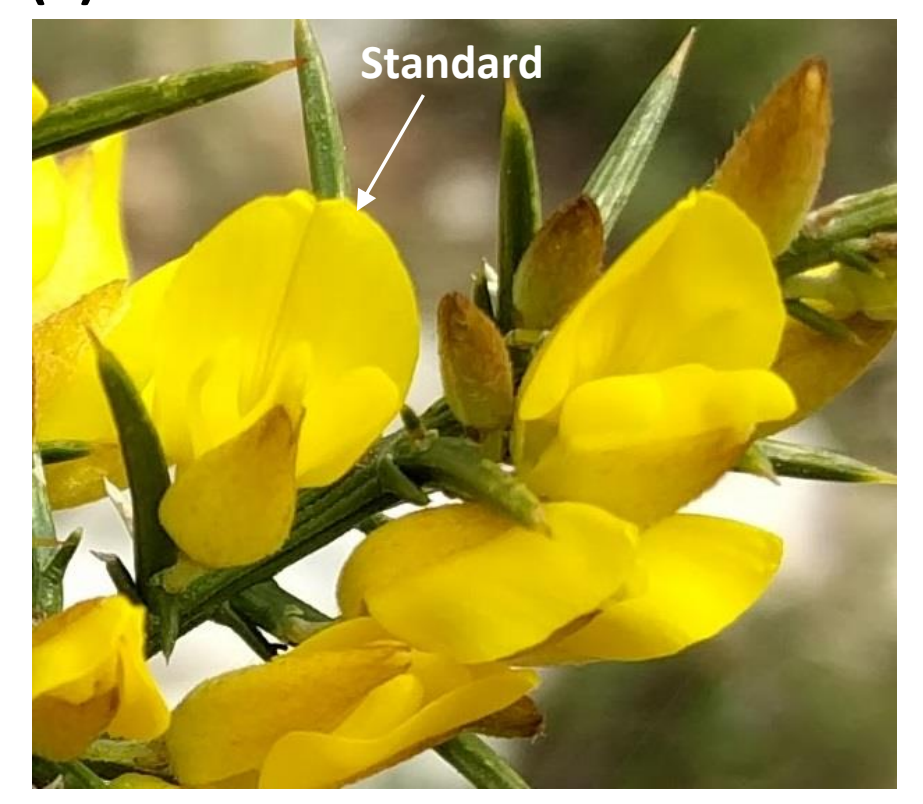

(b)

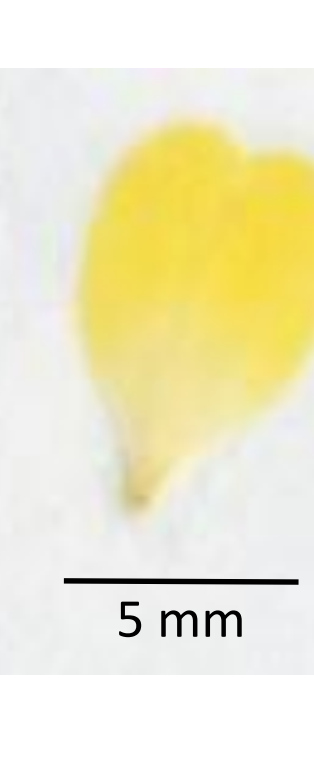

(c)

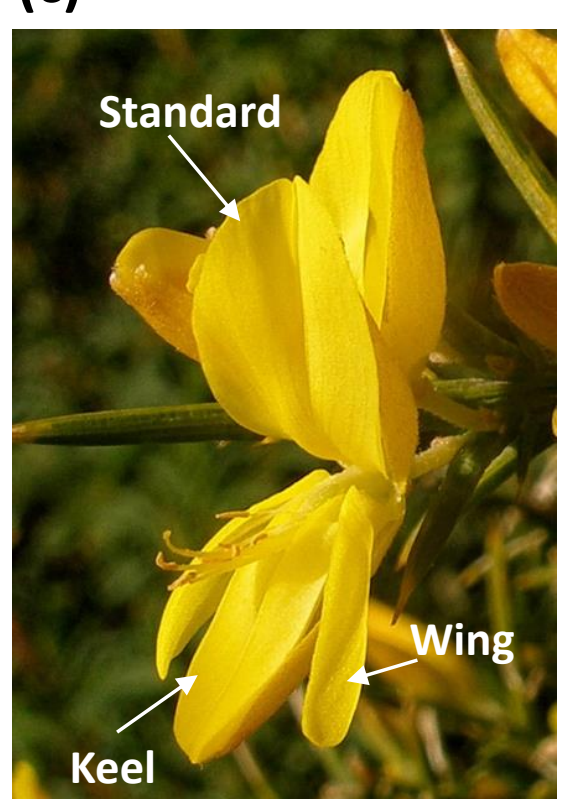

Figure 1 

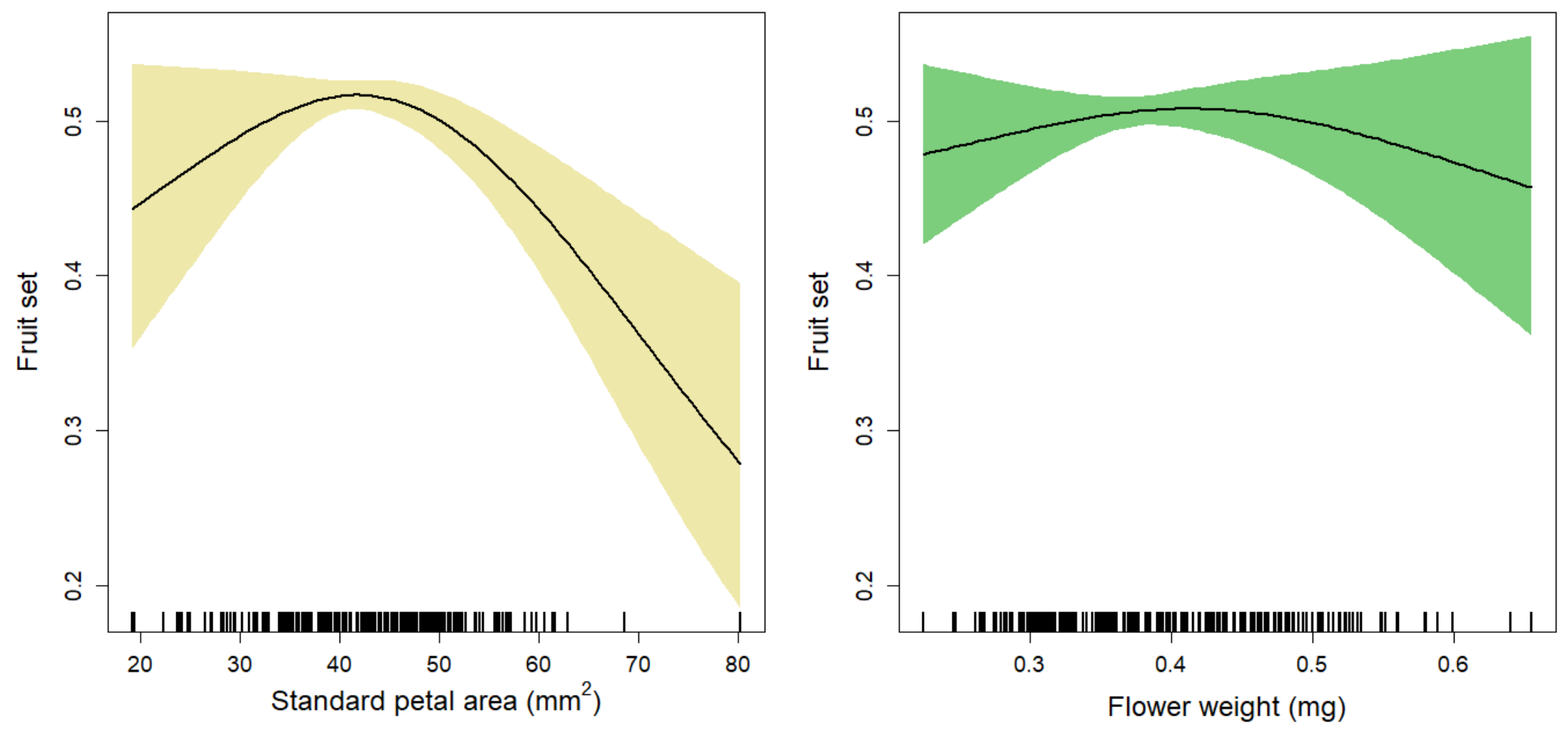

Fig. 2 